\title{
For Effective, Earlier and Simplified Diagnosis of Retinopathy of Prematurity (RoP), a Probe through Digital Image Processing Algorithm in B-Scan
}

\author{
K R N Aswini ${ }^{1}$, S Vijaya Raghavan ${ }^{2}$, Sreekanth NP ${ }^{3}$, Manju Sree RP ${ }^{4}$ \\ ${ }^{1}$ Ph. D Scholar, ${ }^{2}$ Assistant Professor, Department of ECE, SCSVMV Deemed University, Kanchipuram, Chennai, \\ ${ }^{3}$ Chief Medical Officer, ${ }^{4}$ Senior Medical Officer, Sreedhareeyam Ayurvedic Eye Hospital and Research Centre, \\ Koothattukulam, Kerala
}

\begin{abstract}
Eyes are the most vital sensory organ, which stimulates light and helps us to visualize the colorful world around us. Its internal structure is extremely minute and complex with various layers and chambers present. Retina and its layers, is the most important part of an eye that helps in viewing the objects. In certain ailments, if retina gets affected, the vision becomes challenging and may even lead to permanent blindness. One of such vision threatening diseases, affecting the infants is Retinopathy of Prematurity (RoP). Here, various latest, authentic methodologies and tools used for the diagnosis and management of RoP are reviewed and a novel algorithm has been proposed to measure the percentage deviation of retinal health with respect to prior ultrasonic B scan image. Also, to classify the level or stage of RoP based on the location and severity noticed in the ultrasonic B scan images. The proposed architecture and design would be effective and simplified and enable the Ophthalmologists for earlier and accurate diagnosis of RoP in infants.
\end{abstract}

Key words: Retinopathy of Prematurity (RoP), Retinal health, Stage of RoP, Diadic transformation, Ultrasonic B scan images

\section{Introduction}

Sarvendriyanam Nayanam Pradhanam, means Of all senses, eye is the most vital says Ayurveda, which matches with the principles of modern medicine. It is the sensory organ that makes our life bright by stimulating the sense of vision. The anatomy of human eye is very minute and protected well by its own walls and structures. Out of all the eye structures, the retina which is situated at the dorsal side of the eye is responsible to receive the light and converts it into neural signals which are recognized by the brain. The lens situated at the anterior end focuses light on to the retina. In simple terms, the retina can be compared with an image capturing sensor in our latest day's digital cameras. The retina comprises of blood vessels, nerve fibers, rods and cones, etc which are distributed along the 10 different layers. As the retina plays a pivotal role in the image capturing and analysis, any variation in the structure and/or function of the retina and its layers may lead to complicated vision difficulties and may even cause blindness. The major diseases affecting the eye, especially the retina and its layers are generally termed as Retinopathy. Retinopathy of Prematurity (RoP) is one of the most common congenital disease affecting preterm babies and found challenging to prevent artificial blindness.

Retinopathy of Prematurity, formerly known as retrolental fibroplasia (RLF) is a progressive, vasoproliferative, preventable disorder, chiefly observed in preterm new born infants (born within 30 weeks). The infants born with low birth weight (less than $1500 \mathrm{gms}$ ) are also prone for getting affecting with RoP. The changes and the irregularities in the accumulation of blood vessels increase as an individual grow and may end up causing vision threatening abnormalities, if left untreated. Now-a-days, with advancements in the field of medicine and technology, the rate of survival of premature infants is increasing. Simultaneously, RoP has emerged as one of the primary causes of preventable childhood blindness.

However, the complications of the disorder like visual morbidity can be reduced or avoided with an 
early, exact diagnosis followed by an efficient, timely treatment. The presence and prevalence of RoP, if any can be assessed by an Ophthalmologist by performing a simple screening test within a few weeks after the child's birth. With the advent of the latest non-invasive imaging techniques, the evaluation of RoP has become simpler. The plus disease in RoP is characterized by very severe vascular abnormalities, dilation of rational retinal blood vessels, tortuosity or diffusion of blood vessels, etc. The International Committee for RoP classification has stated that the earlier prognosis of RoP can be specifically analysed by identifying the demarcation line or ridge formation in the retinal blood vessels.

The observation of infants dilated pupil with an indirect ophthalmoscope, telescreening using RetCam digital imaging device, Ultrasound scan, Fundus imaging, etc are the most commonly used diagnostic tests for the study of RoP. A few of the latest treatment modalities include low weight gain proportion, WINROP algorithm, indirect laser photocoagulation, cryotherapy, etc.

\section{Materials}

\section{Literature Survey and Outcomes:}

In the cases of RoP, changes and irregularities in the accumulation of blood vessels increase as an individual grow and may end up causing vision threatening abnormalities, if left untreated. Now-a-days, with advancements in the field of bio-medical technology, the rate of survival of premature infants is increasing. Simultaneously, RoP has emerged as one of the primary causes of preventable childhood blindness. However, the complications of the disorder like visual morbidity can be reduced or avoided with an early, exact diagnosis followed by an efficient, timely treatment. The presence and prevalence of RoP, if any can be assessed by an Ophthalmologist by performing a simple screening test within a few weeks after the child's birth. With the advent of the latest non-invasive imaging techniques, the evaluation of RoP has become simpler. The plus disease in RoP is characterized by very severe vascular abnormalities, dilation of rational retinal blood vessels, tortuosity or diffusion of blood vessels, etc.

The amount of tortuosity is evaluated by formulating a One Dimensional Differential Geometrical Curvature Characterization Method. From the identified retinal images, vessel networks and their individual boundaries are extracted as planar curves. After segmentation, the computation of differential curvature is carried out at segment level and also at vessel level for distinctive vessels. The testing and validation of this method has been carried out on the accessible, local and public datasets and it has been observed that the blood vessels with appreciable amount of tortuosity have a notable variation in the curvature, on comparing with the normal blood vessels ${ }^{1}$.

The retinal blood vessel system is analysed and a fully automated model is proposed based on morphological segmentation so that the available binary images help to analyse the blood vessel structure. The regions where the blood vessels are suppression are identified with better response to stimulus and strengthened segmentation that withstands changes in the environment with unfavourable image criterion. So, the data which is a set of 22 images obtained from RetCam 3, comprising the information about the vascular system are anticipated under a lesser contrast. Also, for this model, a comparison in testing and analysis are made with respect to selected segmentation methods, which depends on objective criteria ${ }^{2}$.

Till now, most of the automatic or semiautomatic tools used to diagnose RoP have focused on assessing the Plus Disease, by identifying the vascular abnormalities in the retina. A few has focused on methodologies that help in identifying the stage of the disease. As the principles and tools used in Deep Neural Networks are proven to be efficient in computer vision, medical imaging, it has given a scope to design a system for an automatic diagnosis of RoP. In this work, the architecture of Convolutional Neural Networks has been proposed. For every examination, it features the identification of the presence of RoP and its severity, if it is present. By analyzing the severity and the progression of the disease, RoP cases are is classified as mild and severe cases. In this proposed architecture, a feature aggregate operator connects 2 sub-networks. From the fundus images, the sub network 1 extracts the high level features. Aggregator operator fuses the extracted features from various images and feeds as an input to the sub network 2 , so that the class or stage can be anticipated. RetCam3 is used in imaging, training and evaluating the large data sets. This model is proven to be efficient in demonstrating the classification of RoP disease accurately ${ }^{3}$.

A tool is proposed for easier diagnosis of stage of RoP and also to locate the affected region in the eye. The input images for this proposed tool are taken from RetCam and the output is represented as a reading on 1 
to 9 scales. By analysing the same, the stage and zone of RoP can be ascertained. As this tool is easy to operate, nurses or paramedical staff need not depend on the specialist for assessment of the disease. ${ }^{4}$

In the cases of RoP, the growth and development of retinal blood vessels is cluttered or unsystematically arranged which would result in causing retinal scar or even retinal detachment in some extreme cases. The stage at which the disease has been diagnosed, the tortuosity index helps to assess the asperity of the disease. It is considered that higher the tortuosity index, greater the chances for the diagnosis of plus disease, which would lead to Aggressive Posterior RoP. Also, it is required to have an expertise in Retinopathy and Ophthalmology to diagnose the condition and assess the stage of the disease. As we lack this especially in the rural areas of the developing countries, a lot many children are left unnoticed till they become completely or partially blind. Keeping this end in view, if the gap between the patient and the specialist doctor is reduced, more number of children will be benefitted. Based on the individual's condition and essentiality, the appointments, treatment plans can be scheduled. More people would be made beneficial with this advancement in technology ${ }^{5}$.

Plus disease in the case of RoP usually, an Ophthalmologist, who is specialised in retina related ailments does a clinical examination to the child and assess the parameters through a Fundus image. It represents the vascular structure of the eye, which can be compared with respect to the features of Gold Standard Image, which needs an expertise. In developing countries, most of the rural areas suffer from lack of specialist doctors, where computer aided systems help to diagnose the disease at the earliest. In this paper, Curvature based method has been developed to measure the delineation of retinal blood vessels using COSFIRE filters and calculation of tortuosity, which has found effective with 0.88 sensitivity and 0.94 specificity as a result on its observation of 35 set of images ${ }^{6}$.

The effective segmentation followed by precise visualisation of retina's vascular curvature is important to understand the health of retina in infants suffering from RoP. But, in case of infants the fundus is of low contrast compared to adults, which makes the segmentation difficult by using, the available computer based retinal image analysers. To overcome this, the blood vessel networks can be fused by a guided filter and mathematical morphological techniques can be applied. The modified mathematical morphological closing operation which is being used in post processing technology helps to identify and analyse the thin and small blood vessels in the output after segmentation. The information and values extracted after segmentation helps in the earlier diagnosis of RoP7.

\section{Proposed Methodology \& Instruments for Research:}

The ultrasonic B scan is considered as a diagnostic tool for the assessment of RoP condition. For this study the best quality images captured by Sonomed Escalon's, Master-Vu B-Scan (Model No: MV5600) are used. It is a portable devise, which can be connected to a PC, laptop and can be used as a bed side imaging system also. By activating the probe at different positions on the closed eyes, the miniscule anatomy of the eye can be visualized on the monitor inter-phased with the probe. The $12 \mathrm{MHz}$ frequency handheld, soft touch transducer probe sends the sonic waves with virtually no sound and captures the still images or continuous video clips which can be viewed as separate frames also. As it is sensitive even for smoother touches, more pressure need not be exerted while scanning. Hence, reduces the compression of cornea. Time varying gain control is adopted to trace out near, medium and far gain fields. While performing the brightness based B scan, the amplitude based A scan image can also be visualized simultaneously. Special tools are available for variable zoom, pan and selection of specific area, brightness and contrast control, on-screen annotation, etc. Sectoral scanning method is used where frame rate id $30 \mathrm{fps}$ ( $\max$ ), and gray scale levels are 256. For an improved penetration, acoustic output can be adjusted by achieving the scan depth of $30-60 \mathrm{~mm}$. The possible electronic and clinical resolutios are $0.015 \mathrm{~mm}$ (max) and $0.1 \mathrm{~mm}$ respectively. The captured images are saved as .jpg or .bmp formats and video clips captured are saved as .wmv up to 100 frames. The precision in terms of distance and angle is $0.046 \mathrm{~mm}$ and $1 / 2$ degree respectively.

The software has a license to be installed in multiple computers or laptops and so, more number of ophthalmologists can use a single machine for easier screening. The database of every patient can be entered and saved separately, which enables patient's privacy protection. Its clinical accuracy is $+-0.1 \mathrm{~mm}$, electrical accuracy is $+-0.32 \mathrm{~mm}$, lens database is 1600 and an increment of lens calculation is in $0.25 \mathrm{D}$. The formulas like Binkhorst, Regression II, Holladay, Hoffer Q, 
Haigis, etc are available for the calculation of intraocular lens power. Latkany myopic regressions, latkany hyperopic formula, Aramberri Double K are used for post refractive IOL calculation.

\section{Sample B Scan Images:}

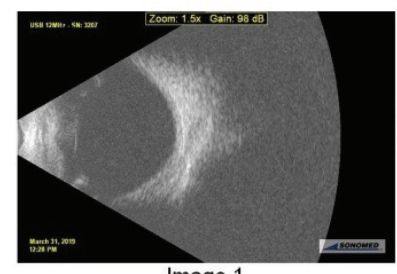

Image 1
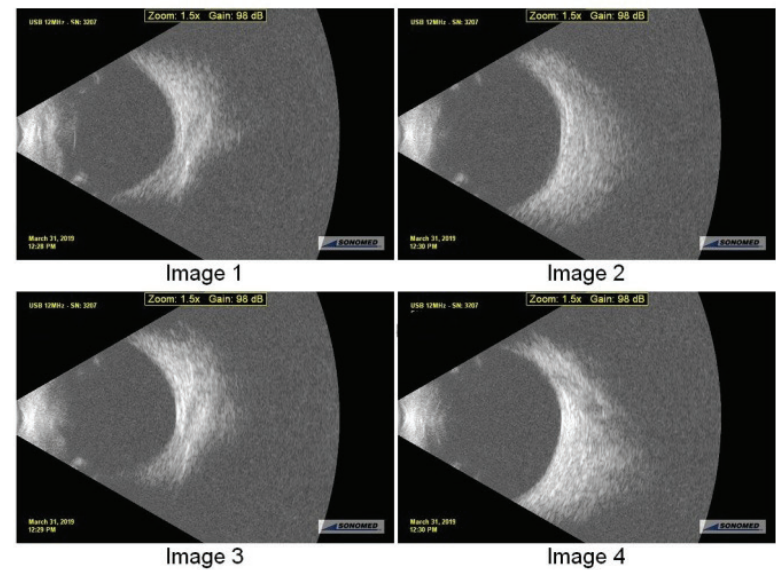

Image 2

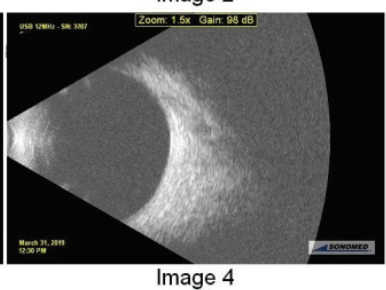

Image A: A few hard Exudates are found in both OD \& OS (Right \& Left Eyes) respectively
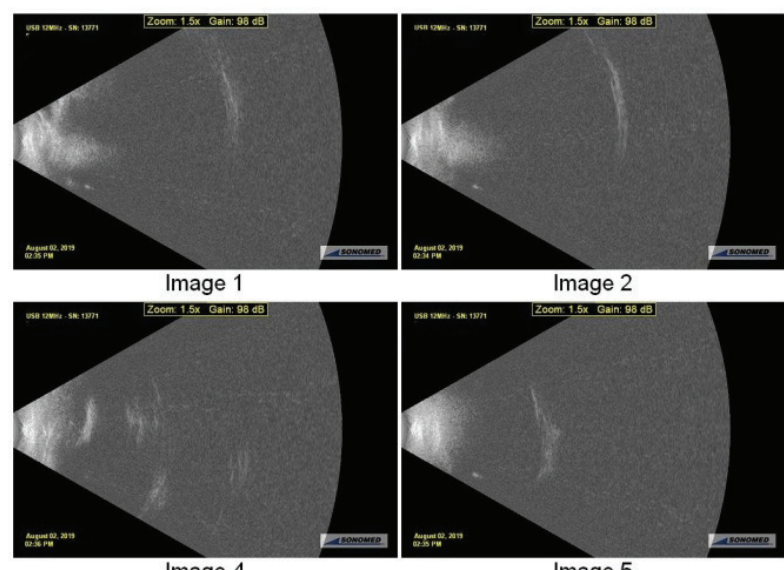

Image 4

Image 5

Image B: The Retinal Structure is Not Formed Completely:
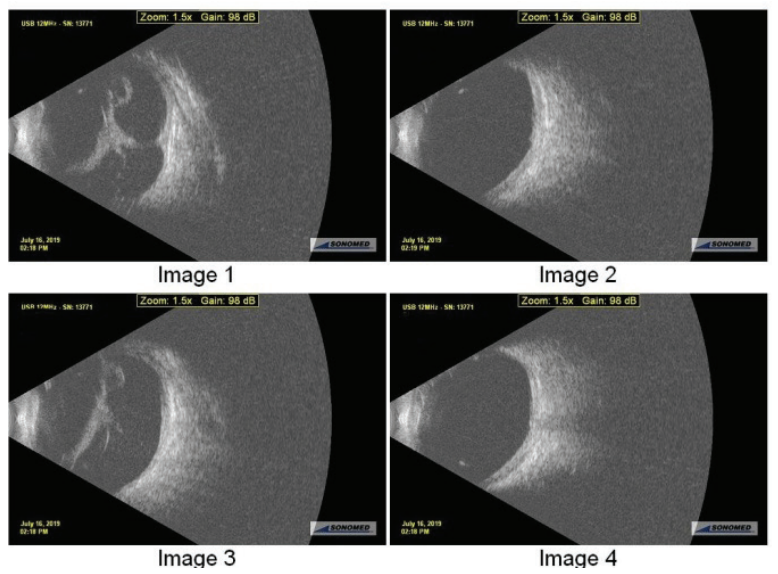

Image C: Symptoms of Retinal Detachment are in OD (right eye) and OS (left eye) is almost norma
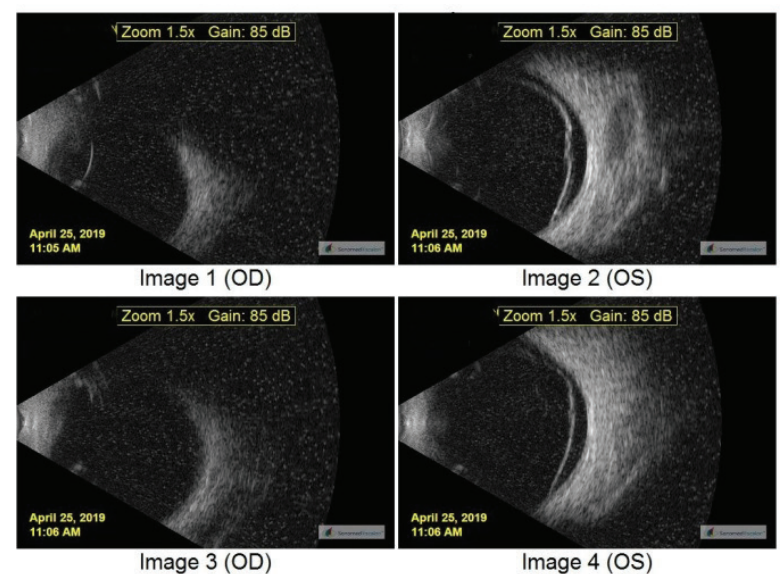

Image D: Tractional retinal Detachment is noticed

\section{Conclusion}

The sample images are subjected to the morphologies in-line with algorithm proposed and found that it has a promising scope in attaining the accurate measurement of the percentage deviation of retinal health with respect to prior Ultrasonic B scan image and also to classify the level or stage of the RoP based on the location and severity noticed in the Ultrasonic B scan. The method would be implemented in real time application so that better diagnostic facility can be provided to infants. The Ophthalmic health of the future generation can be protected for a brighter society.

Acknowledgement: I would like to render my whole hearted gratitude and regards to the management, doctors, Optometry department and Clinical Research team of Sreedhareeyam Ayurvedic Research \& Development Institute, Sreedhareeyam Ayurvedic Eye Hospital and Research Centre, Koothattukulam, Kerala for their constant support and providing me with the data and knowledge bank required as per the research needs. Also, my thankfulness to the Doctors and Members of Institutional Ethics Committee for their acceptance and valuable suggestions, enabling me in taking forward the research work, in-line with the current needs and trends of the society.

Ethical Clearance: The Ethical Clearance is approved by "Institutional Ethics Committee" of Sreedhareeyam Ayurvedic Research \& Development Institute, Koothattukulam, Kerala on 23rd November 2019.

Conflict of Interest: The authors do not have any conflict of interest.

Source of Funding: There is no external agency 
funding for this research. It is self-funding research.

\section{References}

1. Manjunatha R, Koti M, Sheshadri HS. Boundary Extraction and Tortuosity Calculation in Retinal Fundus Images. In: Lecture Notes in Electrical Engineering. Springer Verlag; 2019. p. 1119-30.

2. Krestanova A, Kubicek J, Timkovic J, Penhaker M, Oczka D, Vanus J. Modeling and extraction of retinal blood vessels from RetCam 3 based on morphological segmentation. In: Studies in Computational Intelligence. Springer Verlag; 2020. p. 255-63.

3. Hu J, Chen Y, Zhong J, Ju R, Yi Z. Automated analysis for retinopathy of prematurity by deep neural networks. IEEE Trans Med Imaging. 2019;38(1):269-79.

4. Badarinath D, Chaitra S, Bharill N, Tanveer M, Prasad M, Suma HN, et al. Study of Clinical Staging and Classification of Retinal Images for
Retinopathy of Prematurity (ROP) Screening. In: Proceedings of the International Joint Conference on Neural Networks. 2018.

5. Li T, Martínez López L, Li Y, Institute of Electrical and Electronics Engineers. Proceedings of the 2017 12th International Conference on Intelligent Systems and Knowledge Engineering (ISKE) : IEEE ISKE 2017: November 24-26, 2017, NanJing, JiangSu, China.

6. Sivakumar R, Veena V, John R. A curvature based approach for the automated screening of retinopathy of prematurity in preterm infants. Proc - 13th Int Conf Signal-Image Technol Internet-Based Syst SITIS 2017. 2018;2018-Janua:503-8.

7. Sivakumar R, Eldho M, Jiji C V., Vinekar A, John R. Computer aided screening of retinopathy of prematurity - A multiscale Gabor filter approach. Proc - 2016 6th Int Symp Embed Comput Syst Des ISED 2016. 2017;259-64. 